\title{
Hypoelliptic Degenerate Evolution Equations of the Second Order
}

By

\author{
Masatake MiYAKE*
}

\section{§0. Introduction}

We shall study in this paper the hypoellipticity of the following partial differential operator,

$$
\begin{aligned}
& P\left(x, t ; D_{x}, \partial_{t}\right)=\partial_{t}^{2}+A\left(x, t ; D_{x}\right) \partial_{t}+B\left(x, t ; D_{x}\right), \\
& A\left(x, t ; D_{x}\right)=\sum_{j=0}^{p} a_{j}(x, t) t^{l_{j}} D_{x}^{p-j}, \quad\left(l_{j} \geqq 0, \text { integer }\right), \\
& B\left(x, t ; D_{x}\right)=\sum_{k=0}^{2 p} b_{k}(x, t) t^{m_{k}} D_{x}^{2 p-k}, \quad\left(m_{k} \geqq 0, \text { integer }\right),
\end{aligned}
$$

where $\partial_{t}=\partial / \partial t, D_{x}=-i \partial / \partial x,(x, t) \in R_{x} \times I_{t}, I_{t}=(-1,1)$,

$$
a_{j}(x, t), b_{k}(x, t) \in \mathscr{E}^{\infty}\left(\bar{I}_{t} ; \mathscr{B}_{x}^{\infty}\right)^{1)}, \bar{I}_{t}=[-1,1] \text {. }
$$

For degenerate parabolic differential operators, the study of hypoellipticity has been made by many authors (see [1] [9]). But for degenerate $p$-parabolic differential operators, its study has not been made so detailed (see F. Trèves [10]). So we shall give a sufficient condition for the operator given by $(0.1)$ to be hypoelliptic by constructing very regular parametrices of the operator (see Y. Kato [2], T. Matsuzawa [8]). The operator considered here is a special one, but we want to give an elementary relation between the order of degeneracy of the co-

Communicated by S. Matsuura, April 16, 1975.

* Department of Mathematics, University of Tsukuba, Ibaraki, Japan.

1) $a(x, t) \in \mathscr{E}^{\infty}\left(\bar{I}_{t} ; \mathscr{B}_{x}^{\infty}\right)$ means that $a(x, t) \in \mathscr{B}_{x}^{\infty}$ for any fixed $t \in \bar{T}_{t}$ and infinitely continuously differentiable with respect to $t$ in the usual topology of $\mathscr{B}_{x}^{\infty}$, where $\mathscr{B}_{x}^{\infty}$ $=\left\{u(x) \in C^{\infty}\left(R_{x}\right) ;\left|\partial_{x}^{a} u(x)\right| \leqq M_{\alpha}\right.$ for any $\left.\alpha\right\}$. 
efficient and that of derivative, based on the notion of "modified order" defined in M. Miyake [12]. And for general degenerate $p$-parabolic differential operators of type (0.1), our result in this paper will become a fundamental one (see K. Shinkai [15]).

In Appendix A we shall study the hypoellipticity for the degenerate parabolic differential operator given by

$$
P\left(x, t ; D_{x}, \partial_{t}\right)=\partial_{t}+\sum_{j=0}^{2 m} t^{l_{j}} a_{j}(x, t) D_{x}^{2 m-j}
$$

In Appendix B we shall see that for the study of hypoellipticity of degenerate parabolic differential operators the notion of modified order is a useful one, and we shall be concerned with the operator,

$$
P\left(t ; D_{x}, \partial_{t}\right)=\partial_{t}+a t^{l_{0}} D_{x}^{2 m}+b t^{l_{1}} D_{x}^{2 n},
$$

where $m>n$ and, $a$ and $b$ are non-zero real numbers.

Finally, we note that our result in section 1 can be cxtended to the operator defined in $R_{x}^{n} \times I_{t}$, and it is given in section 8 .

\section{$\S 1 . \quad$ Result}

Our purpose in this paper is to give a sufficient condition for the operator $P\left(x, t ; D_{x}, \partial_{t}\right)$ given by $(0.1)$ to be hypoelliptic. And for this purpose let us assume:

(i) $p$ is a positive even integer,

(ii) $\quad p /\left(l_{0}+1\right) \geqq(p-j) /\left(l_{j}+1\right), \quad j=1,2, \ldots, p$,

(iii) $2 p /\left(m_{0}+2\right) \geqq(2 p-k) /\left(m_{k}+2\right), \quad k=1,2, \ldots, 2 p$,

(iv) $p /\left(l_{0}+1\right)=2 p /\left(m_{0}+2\right)$, that is, $m_{0}=2 l_{0}$,

(v) $\lambda^{2}+a_{0}(x, t) \lambda+b_{0}(x, t)=0$ has simple roots $\lambda=\lambda_{i}(x, t)(i=1,2)$ or a double root $\left(\lambda_{1} \equiv \lambda_{2}\right)$ in $R_{x} \times I_{t}$, and they satisfy that $\operatorname{Re} \lambda_{i}(x, t) \leqq-\delta$ for some positive constant $\delta$.

Then we have

Theorem. Let $P\left(x, t ; D_{x}, \partial_{t}\right)$ be the operator given by $(0.1)$ and let $P$ satisfy the conditions in $(H)$.

(i) If $l_{0}$ is a non-negative even integer, then $P$ and its adjoint ${ }^{t} P$ 
are hypoelliptic in $R_{x} \times I_{t}$.

(ii) If $l_{0}$ is a positive odd integer, then ${ }^{t} P$ is hypoelliptic.

We note that under the conditions in $(H)$ we can easily show that it suffices to consider the following operator instead of $P$ given by (0.1),

$$
\begin{aligned}
& P\left(x, t ; D_{x}, \partial_{t}\right)=\left(\partial_{t}+A\left(x, t ; D_{x}\right)\right)\left(\partial_{t}+B\left(x, t ; D_{x}\right)\right)+C\left(x, t ; D_{x}\right), \\
& A\left(x, t ; D_{x}\right)=\sum_{j=0}^{p} t^{l_{j}} a_{j}(x, t) D_{x}^{p-j}, \\
& B\left(x, t ; D_{x}\right)=\sum_{j=0}^{p} t^{l_{j}} b_{j}(x, t) D_{x}^{p-j} \\
& C\left(x, t ; D_{x}\right)=\sum_{k=1}^{2 p} t^{m_{k}} c_{k}(x, t) D_{x}^{2 p-k}
\end{aligned}
$$

where we may assume without loss of generality that $a_{j}, b_{j}, c_{k} \in \mathscr{E}^{\infty}\left(\bar{I}_{t}\right.$; $\left.\mathscr{B} x_{x}^{\infty}\right)$.

Then the conditions in $(H)$ are transformed into

(i) $p$ is a positive even integer,

(ii) $\quad p /\left(l_{0}+1\right) \geqq(p-j) /\left(l_{j}+1\right), \quad j=1,2, \ldots, p$,

(iii) $\quad p /\left(l_{0}+1\right) \geqq(2 p-k) /\left(m_{k}+2\right), \quad k=1,2, \ldots, 2 p$,

(iv) $\operatorname{Re} a_{0}(x, t) \geqq \delta, \operatorname{Re} b_{0}(x, t) \geqq \delta$ in $R_{x} \times I_{t}$ for some positive constant $\delta$.

In fact, it suffices to check that $(p-j) /\left(l_{j}+1\right) \leqq p /\left(l_{0}+1\right)$ and $(p-k) /$ $\left(l_{k}+1\right) \leqq p /\left(l_{0}+1\right) \quad$ imply $\quad(2 p-j-k) /\left(l_{j}+l_{k}+2\right) \leqq p /\left(l_{0}+1\right)$ and that $\left(\partial_{t}\right.$ $+A)\left(\partial_{t}+B\right)+D\left(x, t ; D_{x}\right) \partial_{t}+C\left(x, t ; D_{x}\right)=\left(\partial_{t}+A+D\right)\left(\partial_{t}+B\right)-D B+C$.

Hence in this paper we shall prove our theorem for the operator $P$ given by (1.1) under the conditions in $(\tilde{H})$. And we shall only prove Theorem-(i), since the proof of Theorem-(ii) is obtained easily combining our argument and that of Y. Kannai [1] or Y. Kato [3]. Therefore throughout this paper we assume that $l_{0}$ is a non-negative even integer.

In section 2 we shall give an outline of the proof of our theorem and sections 3-7 are devoted to the proof of our theorem.

\section{§2. An Outline of the Proof of Theorem}

As mentioned in section 1, we shall prove only Theorem-(i), so we 
assume that $l_{0}$ is a non-negative even integer.

Let $\mathscr{K}(x, y, t, s)$ be a kernel defined in $R_{x} \times R_{y} \times I_{t} \times I_{s}$ defined by

$$
[\mathscr{K} v](x, t)=(2 \pi)^{-1} \int_{-1}^{t} d s \int_{-\infty}^{\infty} e^{i x \xi} K(x, \xi, t, s) \hat{v}(\xi, s) d \xi
$$

where $v(y, s) \in C_{0}^{\infty}\left(R_{y} \times I_{s}\right)$ and $\hat{v}(\xi, s)$ denotes the partial Fourier transform of $v(y, s)$ with respect to $y$, that is,

$$
\hat{v}(\xi, s)=\int_{-\infty}^{\infty} e^{-i y \xi} v(y, s) d y
$$

Then from a formal calculation we can obtain a condition on $K(x, \xi$, $t, s$ ) that $\mathscr{K}$ becomes a fundamental solution of $P$. Indeed,

$$
\begin{aligned}
P_{x, t}[ & \mathscr{K} v](x, t) \\
= & (2 \pi)^{-1} \int_{-1}^{t} d s \int_{-\infty}^{\infty} e^{i x \xi} P\left(x, t ; \xi+D_{x}, \partial_{t}\right) K(x, \xi, t, s) \hat{v}(\xi, s) d \xi \\
& +(2 \pi)^{-1}\left[\int_{-\infty}^{\infty} e^{i x \xi}\left(\partial_{t}+B\left(x, t ; \xi+D_{x}\right)\right) K(x, \xi, t, s) \hat{v}(\xi, s) d \xi\right]_{s=t} \\
& +(2 \pi)^{-1}\left(\partial_{t}+A\left(x, t ; D_{x}\right)\right)\left[\int_{-\infty}^{\infty} e^{i x \xi} K(x, \xi, t, s) \hat{v}(\xi, s) d \xi\right]_{s=t}
\end{aligned}
$$

implies that in order that $\mathscr{K}$ is a fundamental solution of $P$ (i.e. $P[\mathscr{K} v]$ $=v$ ), it must be hold that

$$
\left\{\begin{array}{l}
P\left(x, t ; \xi+D_{x}, \partial_{t}\right) K(x, \xi, t, s)=0 \text { in }-1<s<t<1 \\
\left.K(x, \xi, t, s)\right|_{t=s}=0,\left.\quad \partial_{t} K(x, \xi, t, s)\right|_{t=s}=1, \\
K(x, \xi, t, s)=0 \text { in }-1<t<s<1 .
\end{array}\right.
$$

Now let $\left\{K_{j}(x, \xi, t, s)\right\}_{j=0}^{\infty}$ be a sequence of approximate solutions of (2.2) defined by

$$
\begin{aligned}
& \left\{\begin{array}{l}
P\left(x, t ; \xi, \partial_{t}\right) K_{0}(x, \xi, t, s)=0 \text { in }-1<s<t<1 \\
\left.K_{0}\right|_{t=s}=0,\left.\quad \partial_{t} K_{0}\right|_{t=s}=1, \quad K_{0}=0 \text { in }-1<t<s<1
\end{array}\right. \\
& \left\{\begin{array}{l}
P\left(x, t ; \xi, \partial_{t}\right) K_{j}=-P_{1}\left(x, t, \xi ; D_{x}, \partial_{t}\right) K_{j-1} \text { in }-1<s<t<1, \\
\left.K_{j}\right|_{t=s}=\left.\partial_{t} K_{j}\right|_{t=s}=0, \quad K_{j}=0 \text { in }-1<t<s<1, \quad j=1,2, \ldots
\end{array}\right.
\end{aligned}
$$


where $P_{1}\left(x, t, \breve{\zeta} ; D_{x}, \partial_{t}\right)=P\left(x, t ; \xi+D_{x}, \partial_{t}\right)-P\left(x, t ; \xi, \partial_{t}\right)$.

Then obviously we have

$$
\begin{aligned}
& P_{x, t}\left[\sum_{j=0}^{k} \mathscr{K}_{j}(x, y, t, s) v(y, s)\right]=v(x, t) \\
& \quad+(2 \pi)^{-1} \int_{-1}^{t} d s \int_{-\infty}^{\infty} e^{i x \xi} P_{1}\left(x, t, \xi ; D_{x}, \partial_{t}\right) K_{k}(x, \xi, t, s) \hat{v}(\xi, s) d \xi .
\end{aligned}
$$

for any $v(y, s) \in C_{0}^{\infty}\left(R_{y} \times I_{s}\right)$, where $\mathscr{K}_{j}$ is a kernel defined by oscillatory integral,

$$
\mathscr{K}_{j}(x, y, t, s)=(2 \pi)^{-1} \int_{-\infty}^{\infty} e^{i(x-y) \xi} K_{j}(x, \xi, t, s) d \xi
$$

Hence we have

$$
P_{x, t}\left[\sum_{j=0}^{k} \mathscr{K}_{j}(x, y, t, s)\right]=\delta(x-y, t-s)+F_{k}(x, y, t, s),
$$

where $\left(\sigma F_{k}\right)(x, \xi, t, s)=P_{1}\left(x, t, \xi ; D_{x}, \partial_{t}\right) K_{k}(x, \xi, t, s)$.

Therefore in order to prove the hypoellipticity of ${ }^{t} P$, it suffices to show (see L. Schwartz [13]),

\section{Proposition 2.1.}

(a) $\mathscr{K}_{j}(x, y, t, s)$ are regular in $(x, t)$ and also in $(y, s)$.

(b) $\mathscr{K}_{j}(x, y, t, s) \in C^{\infty}(W), W=\left\{(x, y, t, s) \in R_{x} \times R_{y} \times I_{t} \times I_{s}\right.$;

$$
|x-y|+|t-s|>0\},
$$

(c) For any fixed non-negative integer $N$, there exists $k_{0}$ such that $F_{k}(x, y, t, s) \in C^{N}\left(R_{x} \times R_{y} \times I_{t} \times I_{s}\right)$ for any $k \geqq k_{0}$.

The proof of this proposition is given in section 3 assuming an elementary Proposition concerning $K_{j}(x, \xi, t, s),(j=0,1,2, \ldots)$.

\section{§3. Proof of Proposition 2.1}

An elementary proposition for the proof of Proposition 2.1 is the following, 
Proposition 3.1. Under the assumptions in Proposition 2.1, there exists a positive constant $d_{0}$ satisfying

$$
\begin{aligned}
\left|\partial_{x}^{v} \partial_{\xi}^{\mu} K_{j}(x, \xi, t, s)\right| \leqq & C(t-s)(1+|\xi|)^{-j d_{0}-\mu} \\
& \times \exp \left[-\varepsilon\left(t^{l_{0}+1}-s^{l_{0}+1}\right)|\xi|^{p}\right], \\
\left|\partial_{t_{s} s}^{l+1} \partial_{x}^{v} \partial_{\xi}^{\mu} K_{j}\right| \leqq C(1 & +|\xi|)^{l p-j d_{0}-\mu} \\
& \times \exp \left[-\varepsilon\left(t^{l_{0}+1}-s^{l_{0}+1}\right)|\xi|^{p}\right],
\end{aligned}
$$

in $-1<s \leqq t<1$, for some positive constants $C$ and $\varepsilon$ depending only on $l, v, \mu$ and $j$. We note that the constant $d_{0}$ does not depend on $l, v, \mu$ and $j$.

Remark 3.1. It also holds that

$$
\begin{aligned}
& \partial_{x}^{v} \partial_{\xi}^{\mu} K_{j} \rightrightarrows 0 \quad \text { as } \quad t \backslash s, \\
& \partial_{t, s}^{l+1} \partial_{x}^{v} \partial_{\xi}^{\mu} K_{j} \rightrightarrows 0 \text { as } t \backslash s \quad \text { if } \mu>l p, \\
& \partial_{t, s}^{l} \partial_{x}^{v} \partial_{\xi}^{\mu} K_{j} \rightarrow 0 \text { as } t \searrow s \text { if } l \leqq j
\end{aligned}
$$

where $\rightrightarrows$ means uniform convergence in $x$ and $\xi$. In (3.5) its convergence is uniform in $x$ and $\xi$ when $\xi$ varies in a compact set.

The above proposition and remark imply Proposition 2.1 by the same way as T. Matsuzawa [8]. In fact, let us prove it assuming Proposition 3.1, and Proposition 3.1 will be proved in the following sections.

Proof of Proposition 2.1. At first let us show

Proposition 3.2. The oscillatory integrals

$$
\mathscr{K}_{j}(x, y, t, s)=(2 \pi)^{-1} \int_{-\infty}^{\infty} e^{i(x-y) \xi} K_{j}(x, \xi, t, s) d \xi, \quad j=0,1, \ldots
$$

2) (3.1) implies immediately

$(3.1)^{\prime}\left|\partial_{x}^{\nu} \partial_{\xi}^{\mu} K_{j}\right| \leqq C(1+|\xi|)^{-j d_{0}-\mu-p /(l o+1)} \exp \left[-\varepsilon\left(t^{l o+1}-s^{l o+1}\right)|\xi|^{p}\right]$.

In fact, it suffices to note that $t-s \leqq C \cdot X^{p /(l o+1)} \cdot|\xi|^{-p /(l o+1)},(|\xi|>c>0)$, where $X$ is defined by (4.11).

3) $\partial_{\iota, s}^{\iota}=\partial_{\iota}^{l_{1}} \partial_{s}^{l_{2}},\left(l_{1}+l_{2}=l\right)$. 
belong to $C^{\infty}(W)$, where $W=\left\{(x, y, t, s) \in R_{x} \times R_{y} \times I_{t} \times I_{s} ;|x-y|+|t-s|\right.$ $>0\}$.

Proof. It is obvious that $\mathscr{K}_{j}(x, y, t, s)$ is infinitely differentiable when $t \neq s$. Let us consider

$$
\begin{aligned}
(x-y)^{\mu} \mathscr{K}_{j}(x, y, t, s) & =(2 \pi)^{-1} \int_{-\infty}^{\infty} D_{\xi}^{\mu} e^{i(x-y) \xi} K_{j}(x, \xi, t, s) d \xi \\
& =(2 \pi)^{-1} \int_{-\infty}^{\infty} e^{i(x-y) \xi}\left(-D_{\xi}\right)^{\mu} K_{j}(x, \xi, t, s) d \xi
\end{aligned}
$$

Then from Proposition 3.1 and its remark we see that for any fixed non-ncgative integer $N$, if we chose $\mu$ so that $\mu>N p-j d_{0}+1$, then for any $l, v, \kappa$ such that $l+v+\kappa \leqq N$ we have

$$
\lim _{t \backslash s} \partial_{t, s}^{l} \partial_{x}^{v} \partial_{y}^{\kappa}(x-y)^{\mu} \mathscr{K}_{j}(x, y, t, s)=0
$$

which implics that $(x-y)^{\mu} \mathscr{K}_{j} \in C^{N}\left(R_{x} \times R_{y} \times I_{t} \times I_{s}\right)$.

Q.E.D.

Proposition 3.3. $\mathscr{K}_{j}(x, y, t, s)$ is regular in $(x, t)$ and also in $(y, s)$.

Proof,

(i) The regularity in $(x, t)$ follows immediately from that

$$
\begin{aligned}
& \int_{R_{y} \times I_{s}} \mathscr{K}_{j}(x, y, t, s) v(y, s) d y d s=(2 \pi)^{-1} \int_{-1}^{t} d s \int_{-\infty}^{\infty} e^{i x \xi} \\
& \times K_{j}(x, \xi, t, s) \hat{v}(\xi, s) d \xi \text { for } v(y, s) \in C_{0}^{\infty}\left(R_{y} \times I_{s}\right) .
\end{aligned}
$$

(ii) For the proof of regularity in $(y, s)$, we use the following

Lemma 3.1. (Hörmander [6]) Let $a(x, \xi) \in S^{m}\left(R_{x} \times R_{\xi}\right),{ }^{4)}$ then for any $v(x) \in C_{0}^{\infty}\left(R_{x}\right)$ and any non-negative integer $N$ we have

$$
\left|\int_{-\infty}^{\infty} e^{i x \xi} a(x, \xi) v(x) d x\right| \leqq C_{N}(1+|\xi|)^{m-N}
$$

for some positive constant $C_{N}$.

4) $S^{m}\left(R_{x} \times R_{\xi}\right)\left(=S_{1,0}^{m}\left(R_{x} \times R_{\xi}\right)\right)$ denotes the class of symbols of pseudo-differential operator of order $m$. $S^{-\infty}=\bigcap_{m<\infty} S^{m}$. 
Let us now consider

$$
\begin{aligned}
\int_{-\infty}^{\infty} & \mathscr{K}_{j}(x, y, t, s) u(x, t) d x \\
= & (2 \pi)^{-1} \int_{-\infty}^{\infty} e^{-i y \xi}\left(\int_{-\infty}^{\infty} e^{i x \xi} K_{j}(x, \xi, t, s) u(x, t) d x\right) d \xi \\
= & (2 \pi)^{-1} \int_{-\infty}^{\infty} e^{-i y \xi} F(\xi, t, s) d \xi .
\end{aligned}
$$

Then from Lemma 3.1, $F(\xi, t, s) \in \mathscr{E}^{\infty}\left(\bar{\Delta}_{t, s} ; S^{-\infty}\right),\left(\bar{\Delta}_{t, s}=\{(t, s) ;-1<s \leqq t\right.$ $<1\}$ ) which implies that

$$
\begin{aligned}
& \int_{R_{x} \times I_{t}} \mathscr{K}_{j}(x, y, t, s) u(x, t) d x d t \\
& \quad=(2 \pi)^{-1} \int_{s}^{1} d t \int_{-\infty}^{\infty} e^{-i y \xi} F(\xi, t, s) d \xi \in C^{\infty}\left(R_{y} \times I_{s}\right) .
\end{aligned}
$$

Proposition 3.4. For any fixed non-negative integer $N$, there exists $k_{0}$ such that for any $k \geqq k_{0}$ it holds that

$$
\begin{array}{r}
(2 \pi)^{-1} \int_{-\infty}^{\infty} e^{i(x-y) \xi} P_{1}\left(x, t, \xi ; D_{x}, \partial_{t}\right) K_{k}(x, \xi, t, s) d \xi \\
\in C^{N}\left(R_{x} \times R_{y} \times I_{t} \times I_{s}\right) .
\end{array}
$$

Proof. It is obvious from Proposition 3.1 and its remark.

Summing up the aboves, the proof of Proposition 2.1 is completed. Consequently, the hypoellipticity of ${ }^{t} P$ is obtained. The hypoellipticity of $P$ follows if we repeat the same reasonings for the operator ${ }^{t} P(x$, $\left.-t ; D_{x},-\partial_{t}\right)$.

\section{§4. Study of $K_{0}(x, \xi, t, s)$}

Remember that $K_{0}(x, \xi, t, s)$ is the solution of the equation (2.3). We shall prove Proposition 3.1 for $K_{0}$ dividing many steps.

4.1. Preliminary. At first, let us consider the following ordinary differential equation, 


$$
\left\{\begin{array}{l}
P_{0}\left(x, \xi, t ; \partial_{t}\right) v=\left(\partial_{t}+A(x, t ; \xi)\right)\left(\partial_{t}+B(x, t ; \xi)\right) v=0 \\
\text { in }-1<s<t<1, \\
\left.v\right|_{t=s}=0,\left.\quad \partial_{t} v\right|_{t=s}=1 .
\end{array}\right.
$$

Then the solution of (4.1) is given by

$$
v(x, \xi, t, s)=\int_{s}^{t} \exp \left[\int_{\tau}^{t} B(x, \sigma ; \xi) d \sigma+\int_{s}^{\tau} A(x, \sigma ; \xi) d \sigma\right] d \tau,
$$

in $-1<s \leqq t<1$. Then for $v(x, \xi, t, s)$ we have

Proposition 4.1. Under the assumptions in Theorem-(i) we have

$$
\begin{aligned}
& \left|\partial_{x}^{v} \partial_{\xi}^{\mu} v(x, \xi, t, s)\right| \leqq C(t-s)(1+|\xi|)^{-\mu} \exp \left[-\varepsilon\left(t^{l_{0}+1}-s^{l_{0}+1}\right)|\xi|^{p}\right] \\
& \left|\partial_{t, s}^{l+1} \partial_{x}^{v} \partial_{\xi}^{\mu} v\right| \leqq C(1+|\xi|)^{l^{p-\mu}} \exp \left[-\varepsilon\left(t^{l_{0}+1}-s^{l_{0}+1}\right)|\xi|^{p}\right]
\end{aligned}
$$

for some positive constants $C$ and $\varepsilon$ depending only on $l, v$ and $\mu$. Moreover the facts stated in Remark 3.1 also hold for $v$.

For the proof of this proposition, we prepare the following two lemmas.

Lemma 4.1. (M. Miyake [12]) The conditions that $p /\left(l_{0}+1\right) \geqq(p$ $-j) /\left(l_{j}+1\right),(j=1,2, \ldots, p)$ imply that

$$
\begin{aligned}
& \int_{s}^{t}|\tau|^{l_{j}} d \tau \leqq C\left(t^{l_{0}+1}-s^{l_{0}+1}\right)^{(p-j) / p}, \quad j=1,2, \ldots, p, \\
& \max \left\{(t-s)|t|^{l_{j}},(t-s)|s|^{l_{j}}\right\} \leqq C \int_{s}^{t}|\tau|^{l_{j}} d \tau,
\end{aligned}
$$

for $-1<s \leqq t<1$, where $C$ is a positive constant independent of $t$ and $s$.

Lemma 4.2. Let us assume the conditions in Lemma 4.1 and let $A(x, t ; \xi)$ be given in (4.1). Consider now

$$
E(x ; \xi, t, s)=\exp \left[-\int_{s}^{t} A(x, \tau ; \xi) d \tau\right],-1<s \leqq t<1
$$

then if $\operatorname{Re} a_{0}(x, t) \geqq \delta$ for some positive constant $\delta$, we have 


$$
\left|\partial_{t, s}^{l} \partial_{x}^{v} \partial_{\xi}^{\mu} E\right| \leqq C(1+|\xi|)^{l_{p-\mu}} \exp \left[-\varepsilon\left(t^{l_{0}+1}-s^{l_{0}+1}\right)|\xi|^{p}\right]
$$

Moreover we have

$$
\left|\partial_{t, s}^{l} \partial_{x}^{v} \partial_{\xi}^{\mu} E\right| \rightrightarrows 0 \text { as } t \backslash s \text { when } \mu>l p
$$

Proof. $\partial_{t, s}^{l} \partial_{x}^{v} \partial_{\xi}^{\mu} E$ is expressed by a linear combination of terms,

$$
\begin{aligned}
& E(x, \xi, t, s) \times \prod_{i \in I} \partial_{x}^{v_{i}} \partial_{\xi}^{\mu_{i}} \int_{s}^{t} A(x, \tau ; \xi) d \tau \times \prod_{j \in J} \partial_{t}^{l_{j}} \partial_{x}^{v_{j}} \partial_{\xi}^{\mu_{j}} A(x, t ; \xi) \\
& \quad \times \prod_{k \in K} \partial_{s}^{l_{k}} \partial_{x}^{v_{k}} \partial_{\xi}^{\mu_{k}} A(x, s ; \xi),
\end{aligned}
$$

$\sum_{i} v_{i}+\sum_{j} v_{j}+\sum_{k} v_{k}=v, \sum_{i} \mu_{i}+\sum_{j} \mu_{j}+\sum_{k} \mu_{k}=\mu,|J|+|K| \leqq l$, where $|I|$ denotes the number of elements in $I$. Without loss of generality, it suffices to assume that $|\xi|>c>0$. Let

$$
X=\left(t^{l_{0}+1}-s^{l_{0}+1}\right)^{1 / p}|\xi|
$$

then using the assumptions and Lemma 4.1, we obtain

$$
|E(x, \xi, t, s)| \leqq \text { const. } \exp \left[-c X^{p}+c^{\prime} \sum_{j=1}^{p} X^{p-j}\right] .
$$

On the other hand we have easily that

$$
\begin{aligned}
&\left|\partial_{x}^{v} \partial_{\xi}^{\mu} \int_{s}^{t} A(x, \tau ; \xi) d \tau\right| \leqq \text { const. }(1+|\xi|)^{-\mu}\left\{\sum_{j=0}^{p-\mu} \int_{s}^{t}|\tau|^{l_{j}} d \tau|\xi|^{p-j}\right\} \\
& \leqq \text { const. }{ }^{\prime}(1+|\xi|)^{-\mu}\left\{\sum_{j=0}^{p} X^{p-j}\right\} \\
& \max \left\{\left|\partial_{t}^{l} \partial_{x}^{v} \partial_{\xi}^{\mu} A(x, t ; \xi)\right|,\left|\partial_{s}^{l} \partial_{x}^{v} \partial_{\xi}^{\mu} A(x, s ; \xi)\right|\right\} \leqq C(1+|\xi|)^{p-\mu}
\end{aligned}
$$

Combining from (4.12) to (4.14), we have the desired inequality (4.8).

Finally, (4.9) is now obvious, since from the assumption that $\mu>l p$, in (4.10) there exists at least one of terms of $\partial_{x}^{v_{i}} \partial_{\xi}^{\mu_{i}} \int_{s}^{t} A(x, \tau ; \xi) d \tau$.

Q.E.D.

Proof of Proposition 4.1. Let

$$
G(x, \xi, t, \tau, s)=\exp \left[\int_{\tau}^{t} B(x, \sigma ; \xi) d \sigma+\int_{s}^{\tau} A(x, \sigma ; \xi) d \sigma\right] .
$$


Then from Lemma 4.2, we have easily that

$$
\left|\partial_{x}^{v} \partial_{\xi}^{\mu} G(x, \xi, t, \tau, s)\right| \leqq C(1+|\xi|)^{-\mu} \exp \left[-\varepsilon\left(t^{l_{0}+1}-s^{l_{0}+1}\right)|\xi|^{p}\right]
$$

which implies immediately the inequality (4.3).

In order to prove (4.4), we use the following,

Lemma 4.3. (T. Matsuzawa [8]) Let $f(t, \tau, s) \in C_{t, \tau, s}^{\infty},(s \leqq \tau \leqq t)$, then

$$
\begin{aligned}
\partial_{t}^{p} \partial_{s}^{q}\left(\int_{s}^{t} f(t, \tau, s) d \tau\right) & =\int_{s}^{t} \partial_{t}^{p} \partial_{s}^{q} f(t, \tau, s) d \tau \\
& -\partial_{t}^{p}\left[\sum_{j=1}^{q} C_{j}^{q} \partial_{s}^{q-j} \partial_{\tau}^{j-1} f(t, \tau, s)\right]_{\tau=s} \\
& +\sum_{k=1}^{q} C_{k}^{p}\left[\partial_{t}^{p-k} \partial_{\tau}^{k-1}\left(\partial_{s}^{q} f(t, \tau, s)\right)\right]_{\tau=t},
\end{aligned}
$$

where $C_{s}^{p}=p ! / k !(p-k) !$

Now we remark that we have by the same way as the proof of Lemma 4.2 that

$$
\begin{aligned}
& \left|\partial_{t, \tau, s}^{l} \partial_{x}^{v} \partial_{\xi}^{\mu} G(x, \xi, t, \tau, s)\right| \\
& \quad \leqq C(1+|\xi|)^{l p-\mu} \exp \left[-\varepsilon\left(t^{l_{0}+1}-s^{l_{0}+1}\right)|\xi|^{p}\right]
\end{aligned}
$$

Hence, in order to evaluate $\partial_{t, s}^{l+1} \partial_{x}^{v} \partial_{\xi}^{\mu} v$ it suffices only to consider

$$
\int_{s}^{t} \partial_{t, s}^{l+1} \partial_{x}^{v} \partial_{\xi}^{u} G(x, \xi, t, \tau, s) d \tau
$$

Now without loss of generality we may assume that $\partial_{t, s}^{l+1}=\partial_{t, s}^{l} \partial_{t}$, then $\partial_{t, s}^{l} \partial_{t} \partial_{x}^{v} \partial_{\xi}^{\mu} G(x, \xi, t, \tau, s)$ is expressed by a linear combination of terms,

$$
\partial_{t, s}^{l^{\prime}} \partial_{x}^{v^{\prime}} \partial_{\xi}^{\mu^{\prime}} B(x, t ; \xi) \times \partial_{t, s}^{l-l^{\prime}} \partial_{x}^{v-v^{\prime}} \partial_{\xi}^{\mu-\mu^{\prime}} G, \quad\left(l^{\prime} \leqq l, v^{\prime} \leqq v, \mu^{\prime} \leqq \mu\right) .
$$

Considering (4.17), it suffices only to consider the term,

$$
\int_{s}^{t} \partial_{x}^{v^{\prime}} \partial_{\xi}^{\mu^{\prime}} B(x, t ; \xi) \partial_{t, s}^{l} \partial_{x}^{v-v^{\prime}} \partial_{\xi}^{\mu-\mu^{\prime}} G(x, \xi, t, \tau, s) d \tau
$$

On the other hand, we can show from Lemma 4.1 that 


$$
\begin{aligned}
(t-s)\left|\partial_{x}^{v^{\prime}} \partial_{\xi}^{\mu^{\prime}} B(x, t ; \xi)\right| & \leqq C(t-s)\left(\sum_{j=0}^{p}|t|^{l_{j}}|\xi|^{p-j}\right)(1+|\xi|)^{-\mu^{\prime}} \\
& \leqq C^{\prime}\left(\sum_{j=0}^{p} X^{p-j}\right)(1+|\xi|)^{-\mu^{\prime}}
\end{aligned}
$$

which implies immediately the inequality (4.4).

4.2. Integral equation. We note that the solution $K_{0}(x, \xi, t, s)$ is also the solution of the following integral equation,

$$
\begin{aligned}
& K_{0}(x, \xi, t, s)=v(x, \xi, t, s)-\int_{s}^{t} v(x, \xi, t, \tau) C(x, \tau ; \xi) \\
& \quad \times K_{0}(x, \xi, \tau, s) d \tau .
\end{aligned}
$$

And the solution $K_{0}$ of (4.20) can be approximated as follows,

$$
\begin{aligned}
& K_{0}^{(0)}(x, \xi, t, s)=v(x, \xi, t, s) \\
& K_{0}^{(n+1)}(x, \xi, t, s)=v(x, \xi, t, s) \\
& \quad-\int_{s}^{t} v(x, \xi, t, \tau) C(x, \tau ; \xi) K_{0}^{(n)}(x, \xi, \tau, s) d \tau, \quad n=0,1, \ldots
\end{aligned}
$$

Using (4.16) and (4.17) we shall prove Proposition 3.1.

4.3. Estimate of $\partial_{x}^{v} \partial_{\xi}^{\mu} K_{0}$. For this purpose let us prove

$$
\begin{aligned}
& \left|\partial_{x}^{v} \partial_{\xi}^{\mu}\left\{K_{0}^{(n+1)}(x, \xi, t, s)-K_{0}^{(n)}(x, \xi, t, s)\right\}\right| \\
& \leqq(t-s)(1+|\xi|)^{-\mu} \exp \left[-\varepsilon\left(t^{l_{0}+1}-s^{l_{0}+1}\right)|\xi|^{p}\right] \\
& \quad \times\{M C(t, s, \xi)\}^{n+1}(t-s)^{2(n+1)} / 2(n+1) !
\end{aligned}
$$

$n=0,1,2, \ldots$, for some positive constants $C, M$ and $\varepsilon$, where $C(t, s ; \xi)$ is defined by

$$
C(t, s ; \xi)=\max \left\{\sum_{k=1}^{2 p}|t|^{m_{k}}|\xi|^{2 p-k}, \sum_{k=1}^{2 p}|s|^{m_{k}}|\xi|^{2 p-k}\right\}
$$

In fact, let us show (4.22) by induction on $n$. Since

$$
K_{0}^{(1)}-K_{0}^{(0)}=-\int_{s}^{t} v(x, \xi, t, \tau) C(x, \tau ; \xi) v(x, \xi, \tau, s) d \tau,
$$


$\partial_{x}^{v} \partial_{\xi}^{\mu}\left\{K_{0}^{(1)}-K_{0}^{(0)}\right\}$ is expressed by a linear combination of terms,

$$
\int_{s}^{t} \partial_{x}^{v_{1}} \partial_{\xi}^{\mu_{1}} v \partial_{x}^{v_{2}} \partial_{\xi}^{\mu_{2}} C \partial_{x}^{v_{3}} \partial_{\xi}^{\mu_{3}} v d \tau, \quad v_{1}+v_{2}+v_{3}=v, \mu_{1}+\mu_{2}+\mu_{3}=\mu
$$

Hence using Proposition 4.1 we can obtain the desired inequality (4.22) for $n=0$. We note that $\varepsilon$ and $M$ can be taken independently in $v, \mu$ if $|v|,|\mu| \leqq N$ for fixed $N$. Considering that $K_{0}^{(n)}$ are determined by (4.21), we can easily obtain (4.22) by induction on $n$. (4.22) implies immediately that

$$
\begin{aligned}
& \left|\partial_{x}^{v} \partial_{\xi}^{\mu} K_{0}(x, \xi, t, s)\right| \leqq C(t-s)(1+|\xi|)^{-\mu} \\
& \quad \times \exp \left[-\varepsilon\left(t^{l_{0}+1}-s^{l_{0}+1}\right)|\xi|^{p}+(t-s) \sqrt{M C(t, s ; \xi)}\right] .
\end{aligned}
$$

Lemma 4.4. Under the assumptions in Theorem-(i), it holds that

$$
\max \left\{(t-s)^{2}|t|^{m_{k}},(t-s)^{2}|s|^{m_{k}}\right\} \leqq C\left(t^{l_{0}+1}-s^{l_{0}+1}\right)^{(2 p-k) / p},
$$

$k=1,2, \ldots, 2 p$, for some positive constant $C$.

This is a special case of Lemma 5.2.

Then from Lemma 4.4 we can show that

$$
-\varepsilon\left(t^{l_{0}+1}-s^{l_{0}+1}\right)|\xi|^{p}+C(t-s) \sqrt{C(t, s ; \xi)} \leqq \text { const. }
$$

which implies

$$
\left|\partial_{x}^{v} \partial_{\xi}^{\mu} K_{0}(x, \xi, t, s)\right| \leqq C(t-s)(1+|\xi|)^{-\mu} \exp \left[-\varepsilon\left(t^{l_{0}+1}-s^{l_{0}+1}\right)|\xi|^{p}\right],
$$

for some positive constants $C$ and $\varepsilon$.

4.4. Estimate of $\partial_{z} \partial_{x}^{v} \partial_{\xi}^{\mu} K_{0}$. Differentiating both sides of (4.20) with respect to $t$, we have

$$
\partial_{t} K_{0}=\partial_{t} v-\int_{s}^{t} \partial_{t} v \cdot C \cdot K_{0} d \tau
$$

Now in view of (4.24) and Proposition 4.1, we have

$$
\begin{aligned}
\left|\partial_{t} \partial_{x}^{v} \partial_{\xi}^{\mu} K_{0}\right| \leqq & C(1+|\xi|)^{-\mu} \exp \left[-\varepsilon\left(t^{l_{0}+1}-s^{l_{0}+1}\right)|\xi|^{p}\right] \\
& +C(1+|\xi|)^{-\mu} C(t, s ; \xi)(t-s)^{2} \exp \left[-\varepsilon\left(t^{l_{0}+1}-s^{l_{0}+1}\right)|\xi|^{p}\right]
\end{aligned}
$$


On the other hand, from Lemma 4.4 we have

$$
(t-s)^{2} C(t, s ; \xi) \exp \left[-\varepsilon^{\prime}\left(t^{l_{0}+1}-s^{l_{0}+1}\right)|\xi|^{p}\right] \leqq \text { const., }
$$

which implies

$$
\left|\partial_{t} \partial_{x}^{v} \partial_{\xi}^{\mu} K_{0}\right| \leqq \text { const. }(1+|\xi|)^{-\mu} \exp \left[-\varepsilon\left(t^{l_{0}+1}-s^{l_{0}+1}\right)|\xi|^{p}\right]
$$

for some positive constant $\varepsilon$.

4.5. Estimate of $\partial_{s} \partial_{x}^{v} \partial_{\xi}^{\mu} K_{0}$. Differentiating both sides of (4.20) with respect to $s$, we know that $\partial_{s} K_{0}$ should satisfy the following integral equation,

$$
\begin{aligned}
\partial_{s} K_{0}(x, \xi, t, s)= & \partial_{s} v(x, \xi, t, s)-\int_{s}^{t} v(x, \xi, t, \tau) C(x, \tau ; \xi) \\
& \times \partial_{s} K_{0}(x, \xi, \tau, s) d \tau
\end{aligned}
$$

Then using Proposition 4.1, we can show by the same way as the estimate of $\partial_{x}^{v} \partial_{\xi}^{\mu} K_{0}$ that

$$
\left|\partial_{s} \partial_{x}^{v} \partial_{\xi}^{\mu} K_{0}\right| \leqq C(1+|\xi|)^{-\mu} \exp \left[-\varepsilon\left(t^{l_{0}+1}-s^{l_{0}+1}\right)|\xi|^{p}\right] \text {. }
$$

4.6. Estimate of $\partial_{\varepsilon, s}^{l} \partial_{\varepsilon} \partial_{x}^{v} \partial_{\xi}^{\mu} K_{0}$. At first we note that

$$
(t-s) C(t, s ; \xi) \exp \left[-\varepsilon\left(t^{l_{0}+1}-s^{l_{0}+1}\right)|\xi|^{p}\right] \leqq C(1+|\xi|)^{p} \text {. }
$$

Let operate $\partial_{t, s}^{l} \partial_{t}$ both sides of (4.20), and then using Lemma 4.3, the assumption of induction on $l,(4.25)$ and (4.28) we have easily obtain the desired inequality,

$$
\left|\partial_{t, s}^{l} \partial_{t} \partial_{x}^{v} \partial_{\xi}^{\mu} K_{0}\right| \leqq C(1+|\xi|)^{l p-\mu} \exp \left[-\varepsilon\left(t^{l_{0}+1}-s^{l_{0}+1}\right)|\xi|^{p}\right]
$$

for some positive constants $C$ and $\varepsilon$.

4.7. Estimate of $\partial_{s}^{l+1} \partial_{x}^{v} \partial_{\xi}^{\mu} K_{0}$. Let operate $\partial_{s}^{l+1}$ to both sides of (4.20), then we can show that $\partial_{s}^{l+1} K_{0}$ should satisfy the following integral equation,

$$
\begin{aligned}
\partial_{s}^{l+1} K_{0}(x, \xi, t, s)= & G(x, \xi, t, s)-\int_{s}^{t} v(x, \xi, t, \tau) C(x, \tau ; \xi) \\
& \times \partial_{s}^{l+1} K_{0}(x, \xi, \tau, s) d \tau
\end{aligned}
$$


Then from the assumption of induction we have

$$
\left|\partial_{x}^{v} \partial_{\xi}^{\mu} G(x, \xi, t, s)\right| \leqq C(1+|\xi|)^{l_{p-\mu}} \exp \left[-\varepsilon\left(t^{l_{0}+1}-s^{l_{0}+1}\right)|\xi|^{p}\right]
$$

Hence using (4.31) we can get the desired inequality,

$$
\left|\partial_{s}^{l+1} \partial_{x}^{v} \partial_{\xi}^{\mu} K_{0}\right| \leqq C(1+|\xi|)^{l p-\mu} \exp \left[-\varepsilon\left(t^{l_{0}+1}-s^{l_{0}+1}\right)|\xi|^{p}\right]
$$

for some positive constants $C$ and $\varepsilon$.

Summing up the aboves, Proposition 3.1 for $j=0$ is proved. On the other hand, the facts stated in Remark 3.1 are almost obvious considering (4.9) and the proof of our proposition.

\section{§5. Preliminary for the Study of $K_{j}, j=1,2, \ldots$}

Let us remember that $K_{j},(j=1,2, \ldots)$ is the solution of the equation (2.4). On the other hand, we know that $K_{j}$ is given by

(5.1) $K_{j}(x, \xi, t, s)=-\int_{s}^{t} K_{0}(x, \xi, t, \tau) P_{1}\left(x, \tau, \xi ; D_{\lambda}, \partial_{\tau}\right) K_{j-1}(x, \xi, \tau, s) d \tau$, in $-1<s \leqq t<1$. Where $P_{1}$ is given by

$$
P_{1}\left(x, t, \xi ; D_{x}, \partial_{t}\right)=P\left(x, t ; \xi+D_{x}, \partial_{t}\right)-P\left(x, t ; \xi, \partial_{t}\right)
$$

We shall prove Proposition 3.1 by induction on $j$. Then from the assumption of induction and conditions in $(\tilde{H})$, it suffices to prove the inequalities in Proposition 3.1 when we put the following operators instead of $P_{1}$,

$$
\begin{array}{ll}
a(x, t) t^{l_{j}} \xi^{p-j} \partial_{t}, & p /\left(l_{0}+1\right)>(p-j) /\left(l_{j}+1\right), \\
a(x, t) t^{m_{k}} \xi^{2 p-k}, & p /\left(l_{0}+1\right)>(2 p-k) /\left(m_{k}+2\right),
\end{array}
$$

where $a(x, t) \in \mathscr{E}^{\infty}\left(\bar{I}_{t} ; \mathscr{B}_{x}^{\infty}\right)$.

Hence in the followings we shall study the following two integrals,

$$
H_{j}^{(k)}(x, \xi, t, s)=\int_{s}^{t} K_{0}(x, \xi, t, \tau) \tau^{l_{k}} a(x, \tau) \xi^{p-k} K_{j-1}(x, \xi, \tau, s) d \tau,
$$


where $p /\left(l_{0}+1\right)>(p-k) /\left(l_{k}+1\right), k=1,2, \ldots, p$.

$$
I_{j}^{(k)}(x, \xi, t, s)=\int_{s}^{t} K_{0}(x, \xi, t, \tau) \tau^{m_{k}} a(x, \tau) \xi^{2^{p-k}} K_{j-1}(x, \xi, \tau, s) d \tau,
$$

where $p /\left(l_{0}+1\right)>(2 p-k) /\left(m_{k}+2\right), k=1,2, \ldots, 2 p$.

Let us now give two lemmas which are important to our purpose. Let $R(>1)$ be defined by

$$
\begin{aligned}
R=\max \left\{r ;(p-j) r /\left(l_{j}+1\right) \leqq p /\left(l_{0}+1\right),(p-j) r \leqq p,\right. \\
\left.(2 p-k) r /\left(m_{k}+2\right) \leqq p /\left(l_{0}+1\right),(2 p-k) r \leqq 2 p\right\}
\end{aligned}
$$

Lemma 5.1. Let $R$ be the number defined by (5.7), then

$$
\int_{s}^{t}|\tau|^{l_{j}} d \tau \leqq C\left(t^{l_{0}+1}-s^{l_{0}+1}\right)^{(p-j) R / p}, \quad j=1,2, \ldots, p
$$

for some positive constant $C$ independent on $t$ and $s$.

Its proof is essentially given in M. Miyake [12].

Lemma 5.2. Let $R$ be the number defined by (5.7), then

$$
\max \left\{(t-s)^{2}|t|^{m_{k}},(t-s)^{2}|s|^{m_{k}}\right\} \leqq C\left(t^{l_{0}+1}-s^{l_{0}+1}\right)^{(2 p-k) R / p}
$$

$k=1,2, \ldots, 2 p$, for some positive constant $C$ independent on $t$ and $s$.

Proof. It suffices to prove our lemma when $0 \leqq s \leqq t<1$.

(i) The case where $0 \leqq s \leqq 2 s \leqq t$. From the assumption, we have easily that

$$
(t-s)^{2}|t|^{m_{k}} \leqq t^{m_{k}+2} \quad \text { and } \quad t^{l_{0}+1}-s^{l_{0}+1} \geqq c t^{l_{0}+1}
$$

On the other hand, in view of the determination of $R$, it holds that $m_{k}+2 \geqq\left(l_{0}+1\right)(2 p-k) R / p$ which implies immediately the inequality (5.9). (ii) The case where $0<s<t<2 s$. Since $t^{l_{0}+1}-s^{l_{0}+1}>(t-s) s^{l_{0}}$, it holds that

$$
\left(t^{l_{0}+1}-s^{l_{0}+1}\right)^{(2 p-k) R / p}>\left\{(t-s) s^{l_{0}}\right\}^{(2 p-k) R / p}
$$


Now becausc of that $t^{m_{k}}(t-s)^{2}<c(t-s)^{2} s^{m_{k}}$ and that $(2 p-k) R \leqq 2 p$ and $t-s<s$, it follows

$$
l^{m_{h}}(t-s)^{2} \leqq c\left\{(t-s) s^{l_{0}}\right\}^{(2 p-k) R / p} . s^{\left\{\left(m_{k}+2\right)-\left(l_{0}+1\right)(2 p-k) R\right\} / p} .
$$

From the determination of $R$ we know that $p\left(m_{k}+2\right) \geqq\left(l_{0}+1\right)(2 p-k) R$, hence we get

$$
t^{m_{k}}(t-s)^{2} \leqq c\left\{(t-s) s^{l_{0}}\right\}^{(2 p-k) R / p},
$$

which implies the inequality (5.15).

Q.E.D.

\section{§6. Study of $\left.H_{j}^{(k)}(x, \xi), t, s\right)$}

We shall study $H_{j}^{(k)}$ dividing many steps.

6.1. Estimate of $\partial_{x}^{v} \partial_{\xi}^{\mu} H_{j}^{(k)}$. Recall that $H_{j}^{(k)}$ is given by

$$
H_{j}^{(k)}=\int_{s}^{t} K_{0}(x, \xi, t, \tau) \tau^{l_{k}} a(x, \tau) \xi^{p-k} \partial_{\tau} K_{j-1}(x, \xi, \tau, s) d \tau,
$$

where $p /\left(l_{0}+1\right)>(p-k) /\left(l_{k}+1\right), k=1,2, \ldots, p$.

At first we note that Lemma 5.1 implies that

$$
\max \left\{(t-s)|s|^{l_{k}},(t-s)|t|^{l_{k}}\right\} \leqq C X^{(p-k) R}|\xi|^{-(p-k) R},
$$

where $X$ is defined by (4.11) and $R$ is the number defined by (5.7). Then from the assumption of induction and (6.2) we have

$$
\begin{aligned}
\left|\partial_{x}^{v} \partial_{\xi}^{\mu} H_{j}^{(k)}\right| \leqq & C(t-s)(1+|\xi|)^{-(p-k)(R-1)-(j-1) d_{0}-\mu} \\
& \times \exp \left[-\varepsilon\left(t^{l_{0}+1}-s^{l_{0}+1}\right)|\xi|^{p}\right] .
\end{aligned}
$$

Hence, for any $d_{0}$ such that

$$
0<d_{0}<\min \left\{p /\left(l_{0}+1\right),(p-k)(R-1) ; k=1, \ldots, p-1\right\},
$$

it holds that

$$
\left|\partial_{x}^{v} \partial_{\xi}^{\mu} H_{j}^{(k)}\right| \leqq C(t-s)(1+|\xi|)^{-j d_{0}-\mu} \exp \left[-\varepsilon\left(t^{l_{0}+1}-s^{l_{0}+1}\right)|\xi|^{p}\right]
$$

6.2. Estimate of $\partial_{t} \partial_{x}^{v} \partial_{\xi}^{\mu} \mathbb{H}_{j}^{(k)}$. Differentiating both sides of (6.1) 
with respect to $t$ we have

$$
\partial_{t} H_{j}^{(k)}=\int_{s}^{t} \partial_{t} K_{0} \cdot \tau^{l_{k}} a \xi^{p-k} \partial_{\tau} K_{j-1} d \tau
$$

Hence by the same way as the above we obtain

$$
\left|\partial_{t} \partial_{x}^{v} \partial_{\xi}^{\mu} H_{j}^{(k)}\right| \leqq C(1+|\xi|)^{-j d_{0}-\mu} \exp \left[-\varepsilon\left(t^{l_{0}+1}-s^{l_{0}+1}\right)|\xi|^{p}\right],
$$

for $d_{0}$ defined by (6.3).

6.3. Estimate of $\partial_{s} \partial_{x}^{v} \partial_{\xi}^{\mu} H_{j}^{(k)}$. In this case, we can not obtain the desired inequality from the representation (6.1). So we rewrite it by

$$
\begin{aligned}
H_{j}^{(k)}(x, \xi, t, s)= & -\int_{s}^{t} \partial_{\tau}\left\{K_{0}(x, \xi, t, \tau) \tau^{l_{k}} a(x, \tau)\right\} \xi^{p-k} \\
& \times K_{j-1}(x, \xi, \tau, s) d \tau
\end{aligned}
$$

In order to study (6.6) we divide it into two cases:

(A) The case where $(p-k) R \leqq p /\left(l_{0}+1\right)$. In this case without loss of generality we may put $l_{k}=0$.

(B) The case where $(p-k) R>p /\left(l_{0}+1\right)$.

At first we consider the case (A). In this case (6.6) can be rewritten by

$$
\begin{aligned}
-H_{j}^{(k)}= & \int_{s}^{t} b(x, \tau) K_{0}(x, \xi, t, \tau) \xi^{p-k} K_{j-1}(x, \xi, \tau, s) d \tau \\
& +\int_{s}^{t} a(x, \tau) \partial_{\tau} K_{0}(x, \xi, t, \tau) \xi^{p-k} K_{j-1}(x, \xi, \tau, s) d \tau \\
= & I^{(k)}(x, \xi, t, s)+I I^{(k)}(x, \xi, t, s) .
\end{aligned}
$$

Therefore it suffices only to study the part $I^{(k)}$. Then from the assumption of induction, we have

$$
\begin{array}{r}
\left|\partial_{s} \partial_{x}^{v} \partial_{\xi}^{\mu} I I^{(k)}\right| \leqq C \cdot(t-s)|\xi|^{p-k}(1+|\xi|)^{-(j-1) d_{0}-\mu} \\
\times \exp \left[-\varepsilon\left(t^{l_{0}+1}-s^{l_{0}+1}\right)|\xi|^{p}\right] .
\end{array}
$$

Considering that $(t-s) \leqq C X^{p /\left(l_{0}+1\right)} \cdot|\xi|^{-p /\left(l_{0}+1\right)},(|\xi|>c>0)$, for such $d_{0}$ satisfying 


$$
0<d_{0}<p /\left(l_{0}+1\right)-(p-k)
$$

it follows that

$$
\left|\partial_{s} \partial_{x}^{v} \partial_{\xi}^{\mu} I I^{(k)}\right| \leqq C(1+|\xi|)^{-j d_{0}-\mu} \exp \left[-\varepsilon\left(t^{l_{0}+1}-s^{l_{0}+1}\right)|\xi|^{p}\right]
$$

Next let us consider the case (B). Let

$$
\begin{aligned}
& H_{j}^{(k)}(x, \xi, t, s) \\
& =\int_{s}^{t} \tau^{l_{k}-1} b(x, \tau) \xi^{p-k} K_{0}(x, \xi, t, \tau) K_{J-1}(x, \xi, \tau, s) d \tau \\
& \quad+\int_{s}^{t} \partial_{\tau} K_{0}(x, \xi, t, \tau) \tau^{l_{k}} a(x, \tau) \xi^{p-k} K_{j-1}(x, \xi, \tau, s) d \tau \\
& =I^{(k)}+I I^{(k)} .
\end{aligned}
$$

At first we consider $I^{(k)}$. The following inequality is obtained immediately,

$$
\begin{aligned}
& \left|\partial_{s} \partial_{x}^{v} \partial_{\xi}^{\mu} I^{(k)}\right| \leqq C \max \left\{(t-s)|s|^{l_{k}-1},(t-s)|t|^{l_{k}-1}\right\} \\
& \quad \times|\xi|^{p-k}(1+|\xi|)^{-p /\left(l_{0}+1\right)-(j-1) d_{0}-\mu} \exp \left[-\varepsilon\left(t^{l_{0}+1}-s^{l_{0}+1}\right)|\xi|^{p}\right] .
\end{aligned}
$$

Without loss of generality, we may assume that

$$
p-k>p /\left(l_{0}+1\right) \text {. }
$$

In fact, in the case where $p-k \leqq p /\left(l_{0}+1\right)$ we can easily see that our reasonings in the following are applicable for such $d_{0}$ satisfying $0<d_{0}$ $<p /\left(l_{0}+1\right)$. From the assumption that $(p-k) /\left(l_{k}+1\right)<p /\left(l_{0}+1\right)$ and (6.11), we have

$$
\frac{(p-k)-p /\left(l_{0}+1\right)}{l_{k}}<\frac{p}{l_{0}+1}
$$

Now let

$$
\begin{gathered}
1<R_{0}<\min \left\{R, R^{\prime} ;\left\{(p-k)-p /\left(l_{0}+1\right)\right\} R^{\prime} / l_{k} \leqq p /\left(l_{0}+1\right),\right. \\
\left.\left\{(p-k)-p /\left(l_{0}+1\right)\right\} R^{\prime} \leqq p\right\}
\end{gathered}
$$

then by the same way as the proof of Lemma 5.1 we have 


$$
\begin{aligned}
& \max \left\{(t-s)|s|^{l_{k}-1},(t-s)|t|^{l_{k}-1}\right\} \leqq C \int_{s}^{t}|\tau|^{l_{k}-1} d \tau \\
& \leqq C^{\prime}\left(t^{l_{0}+1}-s^{l_{0}+1}\right)^{\left\{(p-k)-p /\left(l_{0}+1\right)\right\} R_{0} / p} \\
& =C^{\prime} X^{\left\{(p-k)-p /\left(l_{0}+1\right)\right\} R_{0}} \cdot|\xi|^{-\left\{(p-k)-p /\left(l_{0}+1\right)\right\} R_{0}} .
\end{aligned}
$$

Hence for such $d_{0}$ satisfying

$$
0<d_{0}<\left\{(p-k)-\frac{p}{l_{0}+1}\right\}\left(R_{0}-1\right)
$$

it holds that

$$
\left|\partial_{s} \partial_{x}^{v} \partial_{\xi}^{\mu} I^{(k)}\right| \leqq C(1+|\xi|)^{-j d_{0}-\mu} \exp \left[-\varepsilon\left(t^{l_{0}+1}-s^{l_{0}+1}\right)|\xi|^{p}\right]
$$

Next we consider $I I^{(k)}$. In this case we can easily show that for such $d_{0}$ satisfying (6.3)

$$
\left|\partial_{s} \partial_{x}^{v} \partial_{\xi}^{\mu} I I^{(k)}\right| \leqq C(1+|\xi|)^{-j d_{0}-\mu} \exp \left[-\varepsilon\left(t^{l_{0}+1}-s^{l_{0}+1}\right)|\xi|^{p}\right]
$$

Summing up the aboves we have

$$
\begin{array}{r}
\left|\partial_{s} \partial_{x}^{v} \partial_{\xi}^{\mu} H_{j}^{(k)}\right| \leqq C(1+|\xi|)^{-j d_{0}-\mu} \exp \left[-\varepsilon\left(t^{l_{0}+1}-s^{l_{0}+1}\right)|\xi|^{p}\right] \\
k=1,2, \ldots, p .
\end{array}
$$

6.4. Estimate of $\partial_{i, s}^{l+1} \partial_{x}^{v} \partial_{\xi}^{\mu} H_{j}^{(k)}$ In this case, if we repeat the above reasonings we can obtain the desired inequality,

$$
\left|\partial_{t, s}^{l+1} \partial_{x}^{v} \partial_{\xi}^{\mu} H_{j}^{(k)}\right| \leqq C(1+|\xi|)^{l_{p}-j d_{0}-\mu} \exp \left[-\varepsilon\left(t^{l_{0}+1}-s^{l_{0}+1}\right)|\xi|^{p}\right] .
$$

We only remark that for the proof of $(6.18)$ in the case where $\partial_{t, s}^{l+1}$ $=\partial_{s}^{l+1}$ we use (6.6) for the representation of $H_{j}^{(k)}$.

Finally we have to remark that it is easy to see that for $H_{j}^{(k)}$ the same facts stated in Remark 3.1 hold.

\section{§7. Study of $\mathbb{I}_{j}^{(k)}(x, \xi, t, s)$}

Recall that $I_{j}^{(k)}$ is given by

$$
I_{j}^{(k)}=\int_{s}^{t} K_{0}(x, \xi, t, \tau) \tau^{m_{k}} a(x, \tau) \xi^{2 p-k} K_{j-1}(x, \xi, \tau, s) d \tau
$$


where $(2 p-k) /\left(m_{k}+2\right)<p /\left(l_{0}+1\right)$.

Since our desired inequalities are obtained by repeating the arguments in section 6, we only check different points. Lemma 5.2 implies

$$
(t-s)^{2}|\tau|^{m_{k}} \leqq C X^{(2 p-k) R} \cdot|\xi|^{-(2 p-k) R}, \quad s \leqq \tau \leqq t,
$$

where $X$ is defined by (4.11). Hence for such $d_{0}$ satisfying

$$
0<d_{0}<\min \left\{\frac{p}{l_{0}+1},(2 p-k)(R-1) ; k=1,2, \ldots, 2 p-1\right\}
$$

we can obtain the desired inequalities (3.1) and (3.2) for $\partial_{x}^{v} \partial_{\xi}^{\mu} I_{j}^{(k)}$, $\partial_{t} \partial_{x}^{v} \partial_{\xi}^{\mu} I_{j}^{(k)}$ and $\partial_{s} \partial_{x}^{v} \partial_{\xi}^{\mu} I_{j}^{(k)}$.

Let us consider now $\partial_{t, s}^{l+1} \partial_{x}^{v} \partial_{\xi}^{\mu} I_{j}^{(h)}$, and we may assume without loss of generality that $\partial_{t, s}^{l+1}=\partial_{t, s}^{l} \partial_{t}$. Let operate $\partial_{t, s}^{l} \partial_{t}=\partial_{t}^{l_{1}} \partial_{s}^{l-l_{1}} \partial_{t}$ to both sides of (7.1), then we have

$$
\begin{aligned}
\partial_{t, s}^{l} \partial_{t} I_{j}^{(k)}= & \int_{s}^{t} \partial_{t}^{l_{1}+1} K_{0} \tau^{m_{k}} a \xi^{2 p-k} \partial_{s}^{l-1_{1}} K_{j-1} d \tau \\
& -\sum_{i=1}^{l-l_{1}} C_{i}^{l-l_{1}} \partial_{t}^{l_{1}}\left[\partial_{s}^{l-l_{1}-i} \partial_{\tau}^{i-1}\left\{\partial_{t} K_{0} \tau^{m_{k}} a \xi^{2 p-k} K_{j-1}\right\}\right]_{\tau=s} \\
& +\sum_{i=1}^{l 1} C_{i}^{l_{1}}\left[\partial_{t}^{l_{1}-i} \partial_{\tau}^{i-1}\left\{\partial_{t} K_{0} \tau^{m_{k}} a \xi^{2 p-k} \partial_{s}^{l-l_{1}} K_{j-1}\right\}\right]_{\tau=t} \\
= & I^{(k)}+\sum_{i=1}^{l-l_{1}} I I_{i}^{(k)}+\sum_{i=1}^{l_{1}} I I I_{i}^{(k)}
\end{aligned}
$$

In order to study $I^{(k)}$, we divide it into two cases.

(A) The case where $\partial_{t, s}^{l}=\partial_{t}^{l}$. In this case, in view of (7.2) we have for such $d_{0}$ satisfying (7.3)

$$
\left|\partial_{x}^{v} \partial_{\xi}^{\mu} I^{(k)}\right| \leqq C(1+|\xi|)^{l p-j d_{0}-\mu} \exp \left[-\varepsilon\left(t^{l_{0}+1}-s^{l_{0}+1}\right)|\xi|^{p}\right]
$$

(B) The case where $\partial_{t, s}^{l}=\partial_{t}^{l_{1}} \partial_{s}^{l-l_{1}},\left(l-l_{1} \geqq 1\right)$. Considering that $(t-s)|\tau|^{m_{k}} \leqq C X^{(2 p-k) R / 2}|\xi|^{-(2 p-k) R / 2}$, we can also obtained the inequality (7.5) for such $d_{0}$ satisfying

$$
\begin{array}{r}
0<d_{0}<\min \left\{\frac{p}{l_{0}+1}, \frac{(2 p-k)(R-1)}{2}+\frac{k}{2}\right. \\
k=1,2, \ldots, 2 p-1\} .
\end{array}
$$


The study of $I I_{i}^{(k)}$ is more easy, so we omit it. Finally let us consider $I I I_{i}^{(k)}$. In this case, using the following inequality we can get the desired inequality,

$$
\begin{aligned}
\left|t^{m_{k}} \partial_{x}^{v} \partial_{\xi}^{\mu} K_{j-1}\right| \leqq & C(1+|\xi|)^{-(2 p-k) R / 2-(j-1) d_{0}-\mu} \\
& \times \exp \left[-\varepsilon\left(t^{l_{0}+1}-s^{l_{0}+1}\right)|\xi|^{p}\right],
\end{aligned}
$$

$k=1,2, \ldots, 2 p-1$.

Summing up the aboves we have obtained

$$
\left|\partial_{t, s}^{l+1} \partial_{x}^{v} \partial_{\xi}^{\mu} I_{j}^{(k)}\right| \leqq C(1+|\xi|)^{l p-j d_{0}-\mu} \exp \left[-\varepsilon\left(t^{l_{0}+1}-s^{l_{0}+1}\right)|\xi|^{p}\right] .
$$

Finally we have to note that the facts stated in Remark 3.1 also hold for $I_{j}^{(k)}$.

\section{§8. Extension to the Operator Defined in $\mathbb{R}_{x}^{n} \times \mathbb{H}_{\varepsilon}$}

Our theorem stated in section 1 can be extended to the operator defined in $R_{x}^{n} \times I_{t}$,

$$
\begin{aligned}
& P\left(x, t ; D_{x}, \partial_{t}\right)=\partial_{t}^{2}+A\left(x, t ; D_{x}\right) \partial_{t}+B\left(x, t ; D_{x}\right), \\
& A\left(x, t ; D_{x}\right)=\sum_{j=0}^{p} t^{l_{j}} a_{p-j}\left(x, t ; D_{x}\right), a_{p-j}=\sum_{|\alpha|=p-j} a_{\alpha, j}(x, t) D_{x}^{x}, \\
& B\left(x, t ; D_{x}\right)=\sum_{k=0}^{2 p} t^{m_{k}} b_{2 p-k}\left(x, t ; D_{x}\right), b_{2 p-k}=\sum_{|\beta|=2 p-k} b_{\beta, k}(x, t) D_{x}^{\beta},
\end{aligned}
$$

where $\partial_{t}=\partial / \partial_{t}, D_{x}=-i\left(\partial / \partial x_{1}, \ldots, \partial / \partial x_{n}\right), a_{\alpha, j}, b_{\beta, k} \in \mathscr{E}^{\infty}\left(\bar{I}_{t} ; \mathscr{B}_{x}^{\infty}\right)$, and $l_{j}$ and $m_{k}$ are non-negative integers.

Then under the same conditions in section 1 , we can obtain the results in our theorem. In fact, let us assume,

$$
\left\{\begin{array}{l}
\text { (i) } p \text { is a positive even integer, } \\
\text { (ii) } p /\left(l_{0}+1\right) \geqq(p-j) /\left(l_{j}+1\right), \quad j=1,2, \ldots, p, \\
\text { (iii) } 2 p /\left(m_{0}+2\right) \geqq(2 p-k) /\left(m_{k}+2\right), \quad k=1,2, \ldots, 2 p, \\
\text { (iv) } p /\left(l_{0}+1\right)=2 p /\left(m_{0}+2\right), \text { i.e., } m_{0}=2 l_{0},
\end{array}\right.
$$


(v) $\lambda^{2}+a_{p}(x, t ; \xi) \lambda+b_{2 p}(x, t ; \xi)=0$ has simple roots $\lambda=\lambda_{i}(x, t ; \xi)$, $(i=1,2)$ or a double root (i.e. $\left.\lambda_{1} \equiv \lambda_{2}\right)$ in $R_{x}^{n} \times I_{t}$, and they satisfy that $\operatorname{Re} \lambda_{i}(x, t ; \xi) \leqq-\delta|\xi|^{p}$ for some positive constant $\delta$.

Then we have

Theorem 2. Let the operator given by (8.1) satisfy the conditions in (8.2), then

(i) If $l_{0}$ is a non-negative even integer, then $P$ and its adjoint operator ${ }^{t} P$ are hypoelliptic.

(ii) If $l_{0}$ is a positive odd integer, then ${ }^{t} P$ is hypoclliptic.

The proof of the above theorem performed exactly by the same way as in section 2, except the proof of Proposition 3.1. For the proof of Proposition 3.1, we have to consider the following two cases,

(a) the case where $|\xi|$ is bounded,

(b) the case where $|\xi|$ is unbounded.

The later case (b) is not troublesome, since the reasonings in the previous sections are applicable. So we have to check the facts in Remark 3.1 for $K_{0}(x, \xi, t, s)$ when $|\xi|$ is bounded. Now for this purpose, let us transform $K_{0}$ to $v(x, \xi, t, s)$ by

$$
K_{0}(x, \xi, t, s)=\exp \left[-\frac{1}{2} \int_{s}^{t} A(x, \tau ; \xi) d \tau\right] v(x, \xi, t, s)
$$

then we can easily see that $v$ should satisfy the following ordinary differential equation,

$$
\left\{\begin{array}{l}
\partial_{t}^{2} v=F(x, t ; \xi) v \quad \text { in } \quad-1<s<t<1 \\
\left.v\right|_{t=s}=0,\left.\quad \partial_{t} v\right|_{t=s}=1
\end{array}\right.
$$

where

$$
F(x, t ; \xi)=\sum_{k=0}^{2 p} t^{m_{k}} f_{2 p-k}(x, t ; \xi), f_{2 p-k}=\sum_{|\beta|=2 p-k} f_{\beta, k}(x, t) \xi^{\beta}
$$

$p /\left(l_{0}+1\right) \geqq(2 p-k) /\left(m_{k}+2\right), k=0,1, \ldots, 2 p$. We note that in view of the proof of Lemma 4.2 , we have 
Lemma 8.1. If $|\mu|>l p$, then

$$
\partial_{t, s}^{l} \partial_{x}^{v} \partial_{\xi}^{\mu} \exp \left[-\frac{1}{2} \int_{s}^{t} A(x, \tau ; \xi) d \tau\right] \rightarrow 0 \quad \text { as } \quad t \searrow s
$$

where $\rightarrow$ means uniformly convergence in $x$ and $\xi$ when $\xi$ varies in a compact set.

On the other hand, for the solution $v$ of (8.4) it holds

Lemma 8.2. If $|\mu|>l p$, then

$$
\begin{aligned}
& \left|\partial_{t, s}^{t+1} \partial_{x}^{v} \partial_{\xi}^{\mu} v\right| \rightrightarrows 0 \text { as } \quad t \searrow s, \\
& \left|\partial_{x}^{v} \partial_{\xi}^{\mu} v\right| \rightrightarrows 0 \text { as } \quad t \searrow s,
\end{aligned}
$$

where $\rightrightarrows$ indicates the same meaning in Lemma 8.1.

Proof. We can show that $v$ should satisfy the following integral equation,

$$
v(x, \xi, t, s)=(t-s)+\int_{s}^{t}(t-\tau) F(x, \tau ; \xi) v(x, \xi, \tau, s) d \tau
$$

Then in view of the arguments in section 4 , we have

$$
\begin{aligned}
& \left|\partial_{x}^{v} \partial_{\xi}^{\mu} v\right| \leqq C(t-s)(1+|\xi|)^{-\mu} \exp [(t-s) \sqrt{M \cdot C(t, s ; \xi)}] \\
& \left|\partial_{t, s}^{l+1} \partial_{x}^{v} \partial_{\xi}^{\mu} v\right| \leqq C(1+|\xi|)^{l p-\mu} \exp [(t-s) \sqrt{M \cdot C(t, s ; \xi)}]
\end{aligned}
$$

for some positive constants $C$ and $M$, and $C(t, s ; \xi)$ is defined by

$$
C(t, s ; \xi)=\max \left\{\sum_{k=0}^{2 p}|t|^{m_{k}}|\xi|^{2 p-k}, \sum_{k=0}^{2 p}|s|^{m_{k}}|\xi|^{2 p-k}\right\} .
$$

Therefore by induction on $l$, we can prove (8.7) and (8.8). Q.E.D.

Combining the above two lemmas, we can prove the facts stated in Remark 3.1 for $K_{0}$ when $|\xi|$ is bounded.

\section{Appendix A.}

In this appendix we shall give a sufficient condition for the follow- 
ing operator to be hypoelliptic,

$$
P\left(x, t ; D_{x}, \partial_{t}\right)=\partial_{t}+\sum_{j=0}^{2 m} t^{l_{j}} a_{j}(x, t) D_{x}^{2 m-j},
$$

where $(x, t) \in R_{x} \times I_{t}, I_{t}=(-1,1), a_{j} \in \mathscr{E}^{\infty}\left(I_{t} ; \mathscr{B}_{x}^{\infty}\right)$.

For the operator given by (A.1), (or more general operator) the study has been made by many authors and detailed results are obtained. So we only give a result which is not covered in another paper.

Let us assume:

There exists a sequence of integers $\left\{m_{j}\right\}_{j=0}^{k+1}$ satisfying,

(i) $0=m_{0}<m_{1}<\cdots<m_{k}<m_{k+1}=m$,

(ii) $2\left(m-m_{j}\right) /\left(l_{2 m_{j}}+1\right)=\max _{2 m_{j} \leqq i \leqq 2 m_{j+1}-1}(2 m-i) /\left(l_{i}+1\right)$,

(iii) $\operatorname{Re} a_{2\left(m-m_{\jmath}\right)} \geqq \delta>0$ in $R_{x} \times I_{t}, j=0, \ldots, k$.

Then we have

Theorem A. Let the operator $P$ given by (A.1) satisfy the conditions in (A.2), then

(i) If $l_{2 m_{j}},(j=0,1, \ldots, k)$ are even integers, then $P$ and its adjoint operator ${ }^{t} P$ are hypoelliptic.

(ii) If $l_{2 m_{j}},(j=0,1, \ldots, k)$ are odd integers, then ${ }^{t} P$ is hypoelliptic, but $P\left(t ; D_{x}, \partial_{t}\right)$ is not hypoelliptic.

Theorem A is proved by the same way as T. Matsuzawa [8] (see also section 2). Without loss of generality, we assume that $l_{2 m_{j}}$ are even integers. Let $\left\{K_{j}(x, \xi, t, s)\right\}_{j=0}^{\infty}$ be a sequence defined by

$$
\left\{\begin{array}{l}
P\left(x, t ; \xi, \partial_{t}\right) K_{0}(x, \xi, t, s)=0 \quad \text { in }-1<s<t<1, \\
\left.K_{0}\right|_{t=s}=1, \quad K_{0}=0 \quad \text { in }-1<t<s<1
\end{array}\right.
$$

$$
\left\{\begin{array}{l}
P\left(x, t ; \xi, \partial_{t}\right) K_{j}=-P_{1}\left(x, t, \xi ; D_{x}\right) K_{j-1} \quad \text { in } \quad-1<s<t<1 \\
\left.K_{j}\right|_{t=s}=0, \quad K_{j}=0 \quad \text { in } \quad-1<t<s<1, \quad(j=1,2, \ldots)
\end{array}\right.
$$

where $P\left(x, t ; \xi+D_{x}, \partial_{t}\right)=P\left(x, t ; \xi, \partial_{t}\right)+P_{1}\left(x, t, \xi ; D_{x}\right)$. Then for $\left\{K_{j}\right\}_{j=0}^{\infty}$ we have 
Lemma A.1. There exists a positive constant $d_{0}$ such that

$$
\begin{aligned}
\left|\partial_{t, s}^{l} \partial_{x}^{v} \partial_{\xi}^{\mu} K_{j}\right| \leqq & C(1+|\xi|)^{2 m l-j d_{0}-\mu} \\
& \times \exp \left[-\varepsilon \sum_{j=0}^{k}\left(t^{l_{2 m_{j}}+1}-s^{l_{2 m_{j}}+1}\right)|\xi|^{2\left(m-m_{j}\right)}\right]
\end{aligned}
$$

in $-1<s \leqq t<1$, for some positive constants $C$ and $\varepsilon$ depending only on $l, v, \mu$ and $j$. Moreover it holds that

$$
\begin{aligned}
& \partial_{t, s}^{l} \partial_{x}^{v} \partial_{\xi}^{\mu} K_{j} \rightarrow 0 \quad \text { as } \quad t \searrow s \text { if } \mu>2 m l, \\
& \partial_{t, s}^{l} \partial_{x}^{v} \partial_{\xi}^{\mu} K_{j} \rightarrow 0 \quad \text { as } \quad t \searrow s \text { if } 0 \leqq l<j,
\end{aligned}
$$

where the convergence in (A.7) is uniform in $x$ and $\xi$ when $\xi$ varies in a compact set.

Its proof is done by the same way as that of Lemma 4.2. In fact, it suffices to define $X_{j},(j=0,1, \ldots, k)$ by

$$
X_{j}=\left(t^{l_{2 m_{j}}+1}-s^{l_{2 m_{j}}+1}\right)^{1 / 2\left(m-m_{j}\right)} \cdot|\xi|, \quad j=0,1, \ldots, k
$$

instead of $X$ defined by (4.11). So we omit the proof.

\section{Appendix B.}

\section{B. . Introduction}

We shall study in this appendix the hypoellipticity for the following operator,

$$
P\left(t ; D_{x}, \partial_{t}\right)=\partial_{t}+a t^{l_{1}} D_{x}^{2 m}+b t^{l_{2}} D_{x}^{2 n}
$$

where $m>n, l_{i}(i=1,2)$ are non-negative integers and $a$ and $b$ are nonzero real constants.

The study of hypoellipticity for degenerate parabolic operators are concentrated in the point that how the principal part control the lower order terms. That is, if

$$
2 m /\left(l_{1}+1\right) \geqq 2 n /\left(l_{2}+1\right),
$$

then $P$ is hypoelliptic in a neighborhood of $t=0$ iff $\partial_{t}+a t^{l_{1}} D_{x}^{2 m}$ is hypo- 
elliptic. (Y. Kato [3]). And the straight extensions of this result are given in Appendix A. But in the case where

$$
2 m /\left(l_{1}+1\right)<2 n /\left(l_{2}+1\right),
$$

the situation is quite different from the former case. So we shall study the hypoellipticity assuming (B.1.3). Now our results are stated as follows.

Theorem B.1. If $P$ is hypoelliptic in a neighborhood of $t=0$, then $\partial_{t}+b t^{l_{2}} D_{x}^{2 n}$ is hypoelliptic.

Theorem B.2. If $a b>0$ and $a<0$, then $P$ is hypoelliptic.

\section{B.2. Proof of Theorem B.1}

We shall prove by contradiction, so we assume that $\partial_{t}+b t^{l_{2}} D_{x}^{2 n}$ is not hypoelliptic, i.e., we assume that

$$
l_{2} \text { is an odd integer and } b>0 \text {. }
$$

In view of Appendix A, our theorem is obvious when $l_{1}$ is an odd integer and $a>0$, therefore it suffices to consider the following two cases,

Case I. $l_{1}$ is an even integer.

Case II. $l_{1}$ is an odd integer and $a<0$.

At first, let us consider the Case I. Without loss of generality we may assume that $a>0$. In fact, if $a<0$ it suffices only to change the coordinate $t$ by $-t$. Now we remark that from Petrovsky's theorem forward Cauchy problem for $P$ is uniformly well posed in $\mathscr{S}^{\prime}$ in the interval $[0,1]$ and also in $[-1,-\varepsilon]$ for any fixed positive $\varepsilon$. But in the interval $[-1,0]$ it is not uniformly well posed. This fact implies that there exists $f^{-}(x, t) \in \mathscr{E}_{t}^{\infty}\left(\mathscr{S}_{x}\right), t \in[-1,0]$ such that the solution of the Cauchy problem,

$$
\left\{\begin{array}{l}
P u^{-}=f^{-}(x, t) \quad \text { in } \quad(-1,0) \\
\left.u^{-}\right|_{t=-1}=0
\end{array}\right.
$$


satisfies that $u^{-} \in \mathscr{E}_{t}^{\infty}\left(\mathscr{S}_{x}\right),(t \in(-1,0))$ and $u^{-}(x,-0)=\lim _{t>0} u^{-}(x, t) \notin$ $C^{\infty}\left(R_{x}\right)$. Let us now prove this fact. We note that for a given $f^{-}(x, t)$ the solution of (B.2.2) should be given by

$$
\begin{aligned}
u^{-}(x, t)= & \mathscr{F}_{\xi}^{-1}\left(\int _ { - 1 } ^ { t } \operatorname { e x p } \left[-\frac{a}{l_{1}+1}\left(t^{l_{1}+1}-s^{l_{1}+1}\right) \xi^{2 m}\right.\right. \\
& \left.\left.-\frac{b}{l_{2}+1}\left(t^{l_{2}+1}-s^{l_{2}+1}\right) \xi^{2 n}\right] \hat{f}^{-}(\xi, s) d s\right) \\
= & \mathscr{F}_{\xi}^{-1}\left[\hat{u}^{-}(\xi, t)\right]
\end{aligned}
$$

where $\mathscr{F}_{\xi}^{-1}$ denotes the Fourier inverse transform and $\hat{f}^{-}(\xi, t)$ denotes the partial Fourier transform of $f^{-}(x, t)$ with respect to $x$. Now let us define $\hat{f}^{-}(\xi, t)$ by

$$
\hat{f}^{-}(\xi, t)=\left\{\begin{array}{l}
0 \text { when } \xi \leqq 0, t \leqq 0 \\
\exp \left[-\frac{a}{l_{1}+1} t^{l_{1}+1} \xi^{2 m}-\frac{b}{l_{2}+1} t^{l_{2}+1} \xi^{2 n}\right] \\
\quad \times \varphi\left(t \xi^{2 m /\left(l_{1}+1\right)}\right), \text { when } t<0, \xi>0,
\end{array}\right.
$$

where $\varphi(\tau) \in C_{0}^{\infty}\left(R_{\tau}\right), \varphi(\tau) \geqq 0$ and $\operatorname{supp}[\varphi(\tau)] \subset\left[-\frac{1}{2},-\frac{1}{4}\right]$. Considering that $a>0, b>0$ and the assumption (B.1.3), it is easy to show that

$$
\lim _{t>0} \partial_{t}^{j} \partial_{\xi}^{k} \hat{f}^{-}(\xi, t)=0 \text { in } \mathscr{S}_{\xi} \quad \text { for any } j \text { and } k .
$$

On the other hand, it is easy to see that

$$
\hat{u}^{-}(\xi, t) \in \mathscr{E}_{t}^{\infty}\left(\mathscr{S}_{\xi}^{\prime}\right), \quad t \in[-1,0] .
$$

In fact, it suffices to see that

$$
\begin{aligned}
\hat{u}^{-}(\xi, t)=\xi^{-2 m /\left(l_{1}+1\right)} \exp [ & \left.-\frac{a}{l_{1}+1} t^{l_{1}+1} \xi^{2 m}-\frac{b}{l_{2}+1} t^{l_{2}+1} \xi^{2 n}\right] \\
& \times \int_{-\xi^{2 m /\left(l_{1}+1\right)}}^{t \xi^{2 m /\left(l_{1}+1\right)}} \varphi(\tau) d \tau
\end{aligned}
$$

Hence for sufficiently large $\xi>0, \lim _{t>0} \hat{u}^{-}(\xi, t)=\int \varphi(\tau) d \tau \cdot \xi^{-2 m /\left(l_{1}+1\right)}$, which 
shows that $\lim _{t>0} u^{-}(x, t) \notin C^{\infty}\left(R_{x}\right)$. Under the above preparation, let us define $f(x, t), t \in[-1,1]$ by

$$
f(x, t)=\left\{\begin{array}{l}
0, t \geqq 0, \\
f^{-}(x, t), t<0 .
\end{array}\right.
$$

Then the equation $P u=f$ has a solution $u(x, t)$ given by

$$
u(x, t)=\left\{\begin{array}{l}
u^{-}(x, t) \text { in } t \leqq 0 \text { given by (B.2.3) } \\
u(x, t) \text { in } t>0, \text { the solution of } P u=0 \\
\text { with Cauchy data }\left.u\right|_{t=0}=\lim _{t>0} u^{-}(x, t) .
\end{array}\right.
$$

This proves the non-hypoellipticity of $P$.

Let us consider the Case II. In this case also we shall construct $f(x, t) \in \mathscr{E}_{t}^{\infty}\left(\mathscr{S}_{x}\right)$ such that the equation $P u=f$ has a non-smooth solution. Under the assumption of Case II we can easily see that the forward (or the backward) Cauchy problem for $P$ is uniformly well posed in the interval $[-1,-\varepsilon]$ (or $[\varepsilon, 1]$ ) for any fixed positive $\varepsilon$, but not in $[-1,0]$ (or $[0,1]$ ). Hence from the arguments in the above, there exists $f^{-} \in \mathscr{E}_{i}^{\infty}\left(\mathscr{S}_{x}\right), t \in[-1,0]$ (or $f^{+} \in \mathscr{E}_{t}^{\infty}\left(\mathscr{S}_{x}\right), t \in[0,1]$ ) such that the equation $P u^{-}=f^{-}$(or $P u^{+}=f^{+}$) has a solution $u^{-} \in \mathscr{E}_{t}^{\infty}\left(\mathscr{S}_{x}\right), t \in(-1,0)$ $\left(u^{+} \in \mathscr{E}_{t}^{\infty}\left(\mathscr{S}_{x}\right), t \in(0,1)\right)$ but $\lim _{t>0} u^{-} \notin C^{\infty}\left(\lim _{t>0} u^{+} \notin C^{\infty}\right)$. Let us now show that we can choose $f^{+}$and $f^{-}$so that $\lim _{t>0} u^{-}=\lim _{t>0} u^{+}$. Indeed, let $\hat{f}^{-}(\xi, t)$ be given by (B.2.4), then it suffices to give ${ }^{t>0} \hat{f}^{+}(\xi, t)$ by $\hat{f}^{+}(\xi, t)$ $=-\hat{f}^{-}(\xi,-t), t>0$. This implies immediately the non-hypoellipticity of $P$.

Q.E.D.

\section{B.3. Proof of Theorem B.2}

At first we note that under the assumption of Theorem B.2, it follows immediately that the solution of $P u=f \in C^{\infty}(\Omega),\left(0 \in \Omega \subset R_{x, t}^{2}\right)$ belongs to $C^{\infty}\left(\bar{\Omega}_{+}\right),\left(\bar{\Omega}_{+}=\{(x, t) \in \Omega ; t \geqq 0\}\right)$ by the usual method developed in [1] or [3]. But we can not prove that $u \in C^{\infty}\left(\bar{\Omega}_{-}\right),\left(\bar{\Omega}_{-}=\{(x, t) \in \Omega\right.$; $t \leqq 0\}$ ) by the method of fundamental solution. On the other hand, if we can show, 


$$
\lim _{t>0} u(x, t)=u(x,-0) \in \mathscr{D}^{\prime}(\Omega \cap\{t=0\})
$$

then we have $u \in C^{\infty}(\Omega)$. In fact if (B.3.1) is proved, let us decompose $u(x, t)$ as follows (see [13]),

$$
u(x, t)=H(t) u(x, t)+H(-t) u(x, t)+\sum_{j=0}^{N} \delta^{(j)}(t) \otimes v_{j}(x)
$$

for some $v_{j}(x) \in \mathscr{D}^{\prime}\left(I_{x}\right),\left(I_{x} \Subset \Omega \cap\{t=0\}\right)$, where $H(t)$ denotes Heaviside's function. Then

$$
\begin{aligned}
P u= & H(t) P u+H(-t) P u+\delta(t) \otimes\{u(x,+0)-u(x,-0)\}+\sum_{j=0}^{N} \delta^{(j+1)}(t) \otimes v_{j}(x) \\
& +\sum_{j=0}^{N} a t^{l_{1}} \delta^{(j)}(t) \otimes D_{x}^{2 m} v_{j}(x)+\sum_{j=0}^{N} b t^{l_{2}} \delta^{(j)}(t) \otimes D_{x}^{2 n} v_{j}(x)
\end{aligned}
$$

Considering that $P u=f \in C^{\infty}(\Omega)$, it follows that $v_{N-l}(x)=0,(l=0,1, \ldots, N)$. Therefore, $\delta(t) \otimes\{u(x,+0)-u(x,-0)\}=0$, that is, $u(x,+0)=u(x,-0)$. Thus we have $u(x, t) \in \mathscr{E}_{t}^{0}\left(\mathscr{D}^{\prime}\left(I_{x}\right)\right), t \in(-c, c)$ for some positive constant c. Consequently $u(x, t) \in \mathscr{E}_{t}^{\infty}\left(\mathscr{D}^{\prime}\left(I_{x}\right)\right)$, which implies immediately that $u(x, t) \in C^{\infty}\left(I_{x} \times(-c, c)\right)$.

Hence our purpose in the followings is to show (B.3.1). Without loss of generality we may assume that

$$
u(x, t)=\partial_{t}^{j} \partial_{x}^{k} v(x, t), \text { where } v(x, t) \in \mathbb{L}^{1}(\Omega) \text {. }
$$

Since $u \in C^{\infty}\left(\bar{\Omega}_{+}\right)$, we can easily show that

$$
\lim _{t \searrow 0} \partial_{t}^{j-l} \partial_{x}^{k} v(x, t)=v^{(j-l, k)}(x) \in \mathscr{D}^{\prime}\left(I_{x}\right), \quad l=0,1, \ldots, j
$$

Indeed, $\partial_{t}^{j-l} \partial_{x}^{k} v(x, t)$ can be represented by

$$
\begin{aligned}
\partial_{t}^{j-l} \partial_{x}^{k} v(x, t)= & \int_{0}^{t} d t_{1} \int_{0}^{t_{1}} d t_{2} \ldots \int_{0}^{t_{l}-1} u(x, \tau) d \tau \\
& +\sum_{i=1}^{l} v_{i}(x) t^{l-i} /(l-i) !, \text { for some } v_{i} \in \mathscr{D}^{\prime}\left(I_{x}\right),(t>0) .
\end{aligned}
$$

Let us now rewrite $v(x, t)$ as follows,

$$
v(x, t)=\lim _{\varepsilon \downarrow 0}\{H(t-\varepsilon) v+H(-t-\varepsilon) v\} \quad \text { in } \quad \mathscr{D}^{\prime}(\Omega) .
$$


Then we have

$$
\begin{aligned}
P u= & \lim _{\varepsilon \searrow 0}\left\{H(t-\varepsilon) P u+H(-t-\varepsilon) P u+\delta_{\varepsilon}(t) \otimes u(x, \varepsilon)-\delta_{-\varepsilon}(t) \otimes u(x,-\varepsilon)\right. \\
& +\sum_{i=1}^{j} \delta_{\varepsilon}^{(i)}(t) \otimes \partial_{t}^{j-i} \partial_{x}^{k} v(x, \varepsilon)-\sum_{i=1}^{j} \delta_{-\varepsilon}^{(i)}(t) \otimes \partial_{t}^{j-i} \partial_{x}^{k} v(x,-\varepsilon) \\
& +\sum_{i=1}^{j} a t^{l_{1}} \delta_{\varepsilon}^{(i-1)}(t) \otimes \partial_{t}^{j-i} \partial_{x}^{k} D_{x}^{2 m} v(x, \varepsilon) \\
& \left.-\sum_{i=1}^{j} a t^{l_{1}} \delta_{-\varepsilon}^{(i-1)}(t) \otimes \partial_{t}^{j-i} \partial_{x}^{k} D_{x}^{2 m} v(x,-\varepsilon)+\cdots\right\} .
\end{aligned}
$$

On the other hand, we know from (B.3.4) that $\lim _{\varepsilon \succ 0} \partial_{t}^{j-i} \partial_{x}^{k+k^{\prime}} v(x, \varepsilon)$ exists in $\mathscr{D}^{\prime}\left(I_{x}\right)$ for any $k^{\prime} \geqq 0$, therefore

$$
\begin{aligned}
& \lim _{\varepsilon \searrow 0} \sum_{\mu=0}^{j} \delta_{-\varepsilon}^{(\mu)}(t) \otimes\left\{\partial_{t}^{j-\mu} \partial_{x}^{k} v(x,-\varepsilon)+(\text { linear combination of }\right. \\
& \left.\left.\qquad \partial_{t}^{j-\mu^{\prime}} \partial_{x}^{k+k^{\prime}} v(x,-\varepsilon), \mu^{\prime}>\mu\right)\right\}
\end{aligned}
$$

has to exist in $\mathscr{D}^{\prime}$. If we take as test function $\varphi(x, t)$ such a form as $\varphi(x, t)=f(x) g(t)$, where $g(t)=1$ in a neighborhood of $t=0$, we see that

$$
\begin{aligned}
& \lim _{\varepsilon \backslash 0}\left\{\partial_{t}^{j} \partial_{x}^{k} v(x,-\varepsilon)+(\text { linear combination of }\right. \\
& \left.\left.\qquad \partial_{t}^{j-\mu^{\prime}} \partial_{x}^{k+k^{\prime}} v(x,-\varepsilon), \mu^{\prime}>0\right)\right\}
\end{aligned}
$$

exists in $\mathscr{D}^{\prime}\left(I_{x}\right)$. Inductively we can prove that if we take as test function $\varphi(x, t)$ such a form as $\varphi(x, t)=t^{\mu} f(x) g(t)$, where $g(t)=1$ in a neighborhood $t=0$, then

$$
\begin{aligned}
& \lim _{\varepsilon \backslash 0}\left\{\partial_{t}^{j-\mu} \partial_{x}^{k} v(x,-\varepsilon)+(\text { linear combination of }\right. \\
& \left.\left.\qquad \partial_{t}^{j-\mu^{\prime}} \partial_{x}^{k+k^{\prime}} v(x,-\varepsilon), \mu^{\prime}>\mu\right)\right\}
\end{aligned}
$$

exists in $\mathscr{D}^{\prime}\left(I_{x}\right)$, consequently, $\lim _{\varepsilon \backslash 0} \partial_{x}^{k} v(x,-\varepsilon) \in \mathscr{D}^{\prime}\left(I_{x}\right)$. Conversely the above procedure, we can conclude that $\lim _{t>0} u(x, t)$ exists in $\mathscr{D}^{\prime}\left(I_{x}\right)$, which proves (B.3.1).

Q.E.D.

Finally we note that recently R. Rubinstein [14] has studied an operator, 


$$
L=\partial_{t}+t^{n} D_{x}^{2}-t^{m} D_{\lambda}, \quad(n ; \text { even })
$$

He proved that $\mathbb{L}$ is not solvable under the same assumption with (B.1.3), that is, if

$$
\frac{2}{n+1}<\frac{1}{m+1}
$$

then $L$ is not solvable.

\section{References}

[1] Kannai, Y., An unsolvable hypoelliptic differential operator, Israel J. Math., 9 (1971), 306-315.

[2] Kato, Y., The hypoellipticity of degenerate parabolic differential operators, J. Functional Analysis, 7 (1971), 116-131.

[3] - Remarks on hypoellipticity of degenerate parabolic differential operators, Proc. Japan Acad., 47 (1971), 380-384.

[4] Helffer, B., Une classe d'opérateurs hypoelliptique, C. R. Acad. Sci. Paris, 277 (1973), 531-533.

[5] — Sur l'hypoellipticité d'une classe d'opérateurs paraboliques dégénérés, to appear.

[6] Hörmander, L., Pseudo-differential operators and hypoelliptic equations, Amer. Math. Soc. Symp. Pure Math., $\mathbb{1 0}$ (1966), Singular integral operators, 138-183.

[7] - Fourier Integral Operators. I, Acta Math., 127 (1971), 79-183.

[8] Matsuzawa, T., On some degenerate parabolic equations I, Nagoya Math. J., 51 (1973), 57-77.

[9] Taniguchi, K., On the hypoellipticity and the global analytic hypoellipticity of pseudo-differential operators, Osaka J. Math., $\mathbb{1} \mathbb{1}$ (1974), 221-238.

[10] Trèves, F., Concatenations of second order evolution equations applied to local solvability and hypoelliptity, Comm. Pure Appl. Math., 26 (1973), 201250.

[11] Mizohata, S., Hypoellipticité des équations paraboliques, Bull. Soc. Math. France, 85 (1957), 15-50.

[12] Miyake, M., Degenerate parabolic differential equations: Necessity of the well-posedness of the Cauchy problem, J. Math. Kyoto Univ., 14 (1974), 461476.

[13] Schwartz, L., Théorie des distributions, Hermann, Paris. 1966.

[14] Rubinstein, R., Examples of nonsolvable partial differential equations, Trans. Amer. Math. Soc., 199 (1974), 123-129.

[15] Shinkai, K., On the fundamental solution of a degenerate parabolic system, to appear in Proc. Japan Acad. 